\title{
Quantum effects in atomically perfect specular spin valve structures
}

\author{
J.M. Teixeira, J. Ventura, Yu.G. Pogorelov, and J.B. Sousa \\ IFIMUP and Departamento de Física, Universidade do Porto, R. Campo Alegre, 687, Porto, 4169, Portugal
}

\begin{abstract}
A simple tight-binding theoretical model is proposed for spin dependent, current-in-plane transport in highly coherent spin valve structures under specularity conditions. Using quantummechanically coherent and spatially quantized Fermi states in the considered multilayered system, a system of partial Boltzmann kinetic equations is built for relevant subbands to yield the expressions for conductance in parallel or antiparallel spin valve states and thus for the magneto-conductance. It is shown that specularity favors the magnetoresistance to reach its theoretical maximum for this structure close to $100 \%$. This result is practically independent of the model parameters, in particular it does not even need that lifetimes of majority and minority carriers be different (as necessary for the quasiclassical regimes). The main MR effect in the considered limit is due to the transformation of coherent quantum states, induced by the relative rotation of magnetization in the FM layers. Numerical calculation based on the specific Boltzmann equation with an account of spin-dependent specular reflection at the interfaces is also performed for a typical choice of material parameters.
\end{abstract}

PACS numbers: 75.10.Hk; 75.30.Gw; 75.70.Cn; 76.50. $+\mathrm{g}$

\section{INTRODUCTION}

Fabrication of novel nanostructured spintronics devices and the related experimental studies of spin-dependent electron transport stimulate new theoretical approaches to the physical properties of nanosystems where quantum coherence effects can have a decisive role, in contrast to the mostly quasiclassical framework of the traditional electronics. One important class of such systems concerns spin valves [1] formed by two ferromagnetic (FM) layers separated by a thin non-magnetic (NM) spacer. The magnetization of one of the FM layers (called pinned layer) is fixed by the bias from underlying antiferromagnetic (AFM) layer, while the magnetization of the other FM layer (free layer) easily rotates when a small magnetic field is applied. This significantly affects the inplane conductance, leading to relatively high MR values, typical for giant magnetoresistance (GMR) [2], but the technology still demands further improvements. One of them consists in the introduction of nano-oxide layers (NOL's) just above the free layer and inside the pinned layer (so that the pinning is not disrupted) [3]. Such NOL-equipped device, the so called specular spin valve (SSV, Fig. 1b) can more than double the GMR ratio of simpler stacks (Fig. 1 a). The increase of MR is believed to arise from the specular reflection of electrons at the $\mathrm{FM} / \mathrm{NOL}$ interfaces.

But, besides the evident effect of carriers confinement, the reduced normal-to-plane scale $d$ of magnetic layers (few $\mathrm{nm}$ thickness, controlled within a $1 \AA$ precision) might allow for a pronounced quantization of the normal component of quasi-momentum, as already indicated by the recent data on spin-resolved electronic reflection from magnetic nanolayers [4], [5]. Furthermore, it is expected that the relevant modes at the Fermi level for each polarization are dramatically restructured when the mutual polarization of magnetic layers is changed. All this can qualitatively change the kinetics of spin-dependent transport, compared to the usual diffusive scenario for a a)

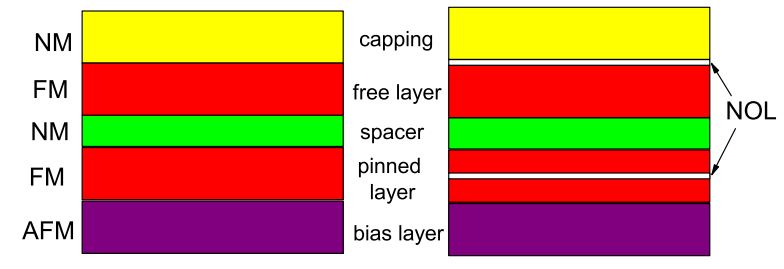

Figure 1: Schematics of spin valve structures: a) common and b) specular.

quasi-continuous spectrum [6]. However, the microscopic understanding of electron specular interface reflection is still far from complete, in particular its role in size quantization and coherence of Fermi states. Here we propose a theoretical description of these effects, through a properly modified Boltzmann kinetic equation, taking into account the formation of transverse-quantized electronic subbands and spin-dependent specular reflection at the interfaces within the simplest tight-binding model, easy enough to advance up to numerical calculations of the MR behavior.

\section{MODEL}

Let us begin from a single metal layer, made of $n$ atomic planes with simple cubic lattice coordination and hopping integral $t$ between nearest neighbors at distance $a$. The respective electronic spectrum for given spin polarization $\sigma=\uparrow, \downarrow$ and planar quasimomentum $\mathbf{k}$ consists of $n$ subbands of the form $\varepsilon_{\alpha, \mathbf{k}, \sigma}=\varepsilon_{\mathbf{k}}+\Delta_{\sigma}+\delta_{\alpha}$ (Fig. 2). Here $\varepsilon_{\mathbf{k}}=2 t\left(2-\cos a k_{x}-\cos a k_{y}\right)$ is the $2 \mathrm{D}$ disper- 


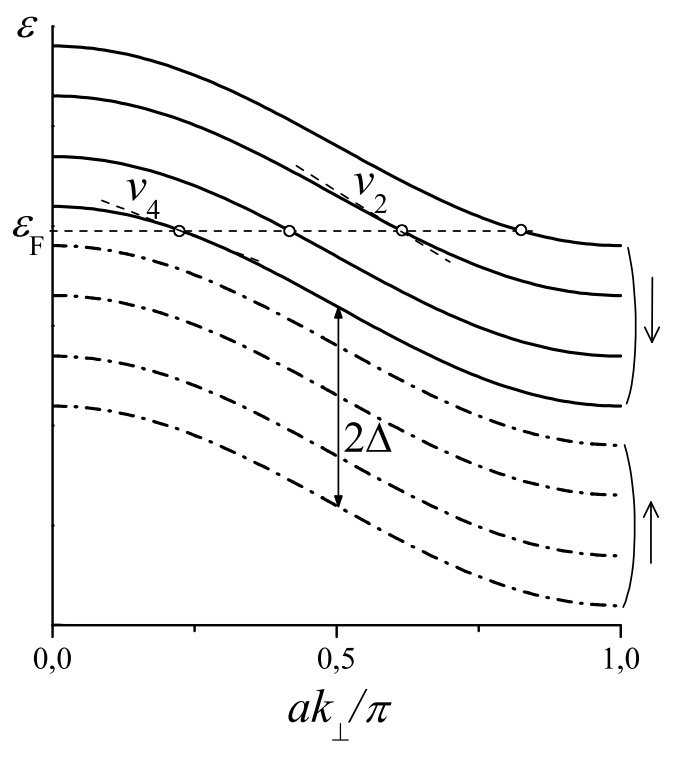

Figure 2: Sketch of the dispersion laws (along the diagonal $k_{x}=k_{y}=k_{\perp}$ of $2 \mathrm{D}$ Brillouin zone) in spin-splitted and spatially quantized subbands of a magnetic nanolayer. The circles indicate Fermi momenta for particular (minority spin) subbands and the related Fermi velocities $v_{\alpha}$ correspond to the slopes of dispersion laws.

sion law for a single plane, and in a ferromagnetic metal it is accompanied by the Stoner energy shift $\Delta_{\sigma}= \pm \Delta$ for minority and majority spins respectively. The spatial quantization is accounted for by the subband shifts $\delta_{\alpha}(\alpha=1, \ldots, n)$ which are the eigenvalues of the $n \times n$ secular equation

$$
\left|\begin{array}{ccccc}
\delta & t & 0 & \ldots & 0 \\
t & \delta & t & \ldots & 0 \\
\ldots & \ldots & \ldots & \ldots & \ldots \\
0 & \ldots & t & \delta & t \\
0 & \ldots & 0 & t & \delta
\end{array}\right|=0
$$

with exact values $\delta_{\alpha}=2 t \cos [\pi \alpha /(n+1)]$. The wave function for the $\alpha, \mathbf{k}, \sigma$ state, at the planar position $\mathbf{r}$ in the $j$-th plane, is $\psi_{\alpha, \mathbf{k}, \sigma}(\mathbf{r}, j)=A_{j}^{(\alpha)} \mathrm{e}^{i \mathbf{k} \cdot \mathbf{r}} \chi_{\sigma}$, where the components of the $n$-dimensional eigenvector $A^{(\alpha)}$ related to the eigenvalue $\delta_{\alpha}$ are explicitly given by

$$
A_{j}^{(\alpha)}=\sqrt{\frac{2}{n+1}} \sin \frac{\pi \alpha j}{n+1},
$$

and $\chi_{\sigma}$ is the spin function.

Next the model is extended to include the hopping $t^{\prime}$ between the neighbor FM and NM layers, hybridizing the subbands of the free FM layer (composed of $n_{f}$ atomic planes) $\varepsilon_{\alpha, \mathbf{k}, \sigma}^{f}$, of the NM spacer (composed of

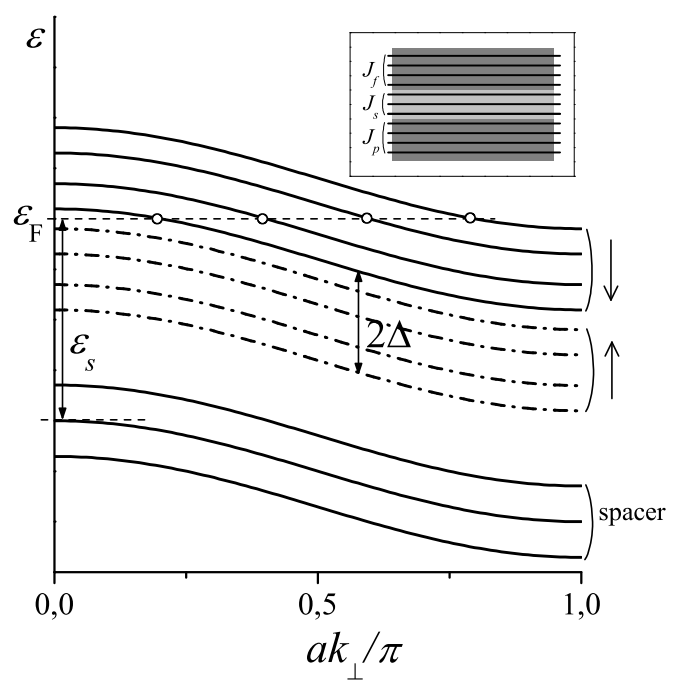

Figure 3: Energy band structure in the trilayered system. All the modes are doubly degenerate and the relevant modes at the Fermi level are marked with circles. Inset: spatial composition of atomic planes forming the sets $J_{f, s, p}$ in $f_{-}, s_{-}$, and $p$-layers.

$n_{s}$ planes) $\varepsilon_{\alpha, \mathbf{k}}^{s}$ (with $\Delta=0$ ), and of the pinned FM layer ( $n_{p}$ planes) $\varepsilon_{\alpha, \mathbf{k}, \sigma}^{p}$. We shall denote the respective eigenvectors (for the uncoupled layers) by $F^{(\alpha)}, S^{(\alpha)}$, and $P^{(\alpha)}$, with the components given again by Eq. 2 for $n=n_{f}, n_{p}, n_{s}$, while the notation $M^{(\alpha)}$ is adopted for the eigenvectors of the coupled system. The specularity effect in this approach is modeled by zero coupling of the FM layers to their outer neighbors. The resulting spectrum totals up to $n_{t}=2\left(n_{f}+n_{s}+n_{p}\right)$ spin-resolved modes with energies $\varepsilon_{\alpha, \mathbf{k}}$ and wave functions $\Psi_{\alpha, \mathbf{k}}(\mathbf{r}, j)=M_{j}^{(\alpha)} \mathrm{e}^{i \mathbf{k} \cdot \mathbf{r}} \chi_{\sigma(\alpha)}$, where $\alpha=1, \ldots, n_{t}$ and $\sigma(\alpha)$ is the implicit polarization of $\alpha$-th mode (Fig. 3). We emphasize that from the total of $n_{t}$ modes, only a smaller number, $n_{r}$, of modes, those present on the Fermi level, are relevant for conductance. Thus, for the characteristic case of FM Co layers, only minority spin subbands should take part in the transport (as suggested by the bulk Co band structure [9]). Moreover, we have to take into account the sizeable differences in the corresponding Fermi velocities $v_{\alpha}$ (practically coincident with those in the uncoupled layers, Fig. 22). The most essential effect of hybridization is on the amplitudes $M_{j}^{(\alpha)}$ which are generally some weighted combinations of all the $F, P, S$ modes, and the crucial point is that the weights of $F, P$ components in the relevant modes are strongly dependent on the mutual polarization of FM layers (see below).

Then the kinetics of the composite system is described by the set of $n_{r}$ distribution functions $f_{\alpha, \mathbf{k}}=f_{\alpha, \mathbf{k}}^{(0)}+g_{\alpha, \mathbf{k}}$ where $f_{\alpha, \mathbf{k}}^{(0)}=\left[\mathrm{e}^{\beta\left(\varepsilon_{\alpha, \mathbf{k}}-\varepsilon_{\mathrm{F}}\right)}+1\right]^{-1}$ is the usual equilibrium 


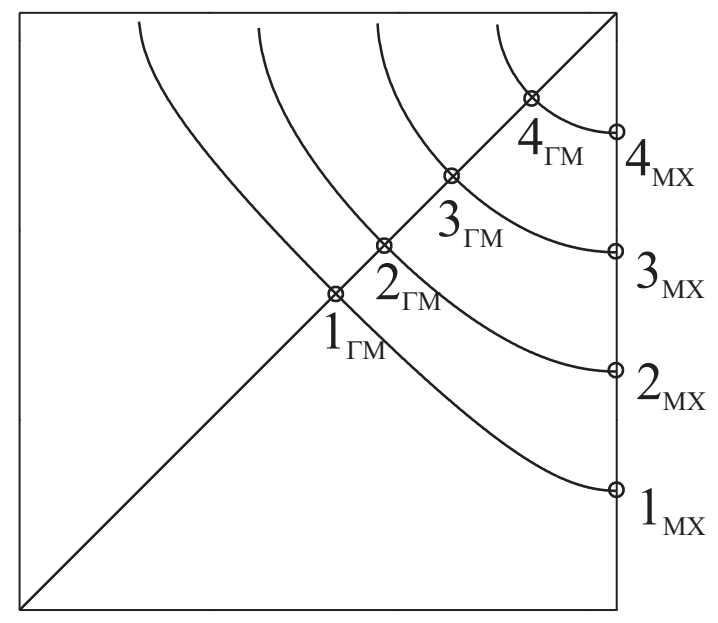

Figure 4: Configurations of Fermi lines for spatially quantized subbands of minority electrons in the Brillouin zone. The characteristic points along high symmetry directions $\Gamma M$ and $M X$ were used to approximate the averages of $v_{\alpha}^{-1}$ and $v_{\alpha}^{2}$.

Fermi function with $\beta=1 / k_{\mathrm{B}} T$ and $g_{\alpha, \mathbf{k}}$ is the nonequilibrium part due to the external electric field $\mathbf{E}$. The current density is given by the sum

$$
\mathbf{j}=\frac{e}{n a} \sum_{\alpha}^{\prime} \int \frac{d \mathbf{k}}{(2 \pi)^{2}} \mathbf{v}_{\alpha, \mathbf{k}} g_{\alpha, \mathbf{k}}
$$

where $\sum^{\prime}$ means summation over the $n_{r}$ relevant modes, $\mathbf{v}_{\alpha, \mathbf{k}}=\hbar^{-1} \partial \varepsilon_{\alpha, \mathbf{k}} / \partial \mathbf{k}$ is the electron velocity, and the components of the non-equilibrium distribution are defined from the system of Boltzmann equations:

$$
\frac{e \mathbf{E}}{\hbar} \cdot \frac{\partial f_{\alpha, \mathbf{k}}^{(0)}}{\partial \mathbf{k}}+\sum_{\beta}^{\prime \prime} \int \frac{a^{2} d \mathbf{k}^{\prime}}{(2 \pi)^{2}} \omega_{\alpha, \mathbf{k}}^{\beta, \mathbf{k}^{\prime}}\left(g_{\beta, \mathbf{k}^{\prime}}-g_{\alpha, \mathbf{k}}\right)=0 .
$$

Here $\sum^{\prime \prime}$ means summation over relevant modes with conserved spin, $\sigma(\alpha)=\sigma(\beta)$, and $\omega_{\alpha, \mathbf{k}}^{\beta, \mathbf{k}^{\prime}}$ is the transition rate due to scattering from $\mathbf{k}$ state of $\alpha$-th subband to $\mathbf{k}^{\prime}$ state of $\beta$-th subband. We consider transitions only due to elastic scattering by random point-like impurities with potential $V$ and concentration $c \ll 1$ (per unit cell). Then the Fermi Golden Rule transition rates are $\omega_{\alpha, \mathbf{k}}^{\beta, \mathbf{k}^{\prime}}=$ $\Omega_{\alpha, \beta} \delta\left(\varepsilon_{\alpha, \mathbf{k}}-\varepsilon_{\beta, \mathbf{k}^{\prime}}\right)$ with the scattering factors (averaged in impurity positions)

$$
\Omega_{\alpha, \beta}=\frac{2 \pi c V^{2}}{\hbar n} \sum_{j}\left|M_{j}^{(\alpha)} M_{j}^{(\beta)}\right|^{2}
$$

In this simple model, the first term in the collision integral of Eq. 4 turns out to be proportional to $\int d \mathbf{k} g_{\beta, \mathbf{k}} \delta\left(\varepsilon_{\mathrm{F}}-\varepsilon_{\beta, \mathbf{k}}\right)$, that is to the average of the nonequilibrium distribution over the Fermi surface and so it should vanish. Then the solution takes the common form $g_{\alpha, \mathbf{k}}=\hbar^{-1} \tau_{\alpha} e \mathbf{E} \cdot \partial f_{\alpha, \mathbf{k}}^{(0)} / \partial \mathbf{k}$ where the relaxation time for the $\alpha$-th mode is defined by

$$
\tau_{\alpha}^{-1}=\sum_{\beta}^{\prime \prime} \rho_{\beta} \Omega_{\alpha, \beta},
$$

including the Fermi densitiy of states $\rho_{\beta}=$ $(a / 2 \pi)^{2} \int d \mathbf{k} \delta\left(\varepsilon_{\beta, \mathbf{k}}-\varepsilon_{\mathrm{F}}\right)$ for each $\beta$-th mode. Then the total conductivity is found from Eq. 3 as a sum of partial contributions:

$$
\sigma_{t o t}=\sum_{\alpha}^{\prime} \sigma_{\alpha}, \quad \sigma_{\alpha}=\frac{e^{2} \tau_{\alpha} \rho_{\alpha}\left\langle v_{\alpha}^{2}\right\rangle}{n a^{3}},
$$

where $\left\langle v_{\alpha}^{2}\right\rangle \approx \rho_{\alpha}^{-1}(a / 2 \pi)^{2} \int d \mathbf{k} v_{\alpha, \mathbf{k}}^{2} \delta\left(\varepsilon_{\alpha, \mathbf{k}}-\varepsilon_{\mathrm{F}}\right)$ is the average of the respective squared Fermi velocity. In fact, this is a particular case of the general Landauer formula [10], written for the present system of $n_{r}$ coherent quantum channels.

The system, Eqs. 116, can be routinely treated by numerical methods at any relative orientation of magnetizations in $f$ - and $p$ - layers, from parallel $(\uparrow \uparrow)$ to antiparallel $(\uparrow \downarrow)$, to result in the principal quantity of interest, the magnetoresistance

$$
\frac{\Delta R}{R}=\frac{\sigma_{t o t}^{\uparrow \uparrow}}{\sigma_{\text {tot }}^{\uparrow \downarrow}}-1=\frac{\sum_{\alpha}{ }^{\prime} \rho_{\alpha}\left\langle v_{\alpha}^{2}\right\rangle \tau_{\alpha}^{\uparrow \uparrow}}{\sum_{\alpha}{ }^{\prime} \rho_{\alpha}\left\langle v_{\alpha}^{2}\right\rangle \tau_{\alpha}^{\uparrow \downarrow}}-1 .
$$

But some qualitative conclusions about the specularity effect on MR in a nanolayered device can be drawn already from simple inspection of the discrete structure of the amplitudes $M_{j}^{(\alpha)}$, according to the following remarks.

First of all, we suppose that in absence of hybridization the majority and minority subbands are well separated from each other and from the spacer subbands (like the situation in bulk $\mathrm{Co}$ and $\mathrm{Cu}$ ). Then we notice that the $j$-configurations of the above amplitudes are essentially different for $\uparrow \uparrow$ and $\uparrow \downarrow$ cases and hence consider them separately. Finally, an important factor for the very existence of GMR (in this quantum coherent conductance regime) is the presence of certain "resonances" between relevant modes at the Fermi level. Namely, a resonance appears between two (unhybridized) modes $\varepsilon_{\alpha, \mathbf{k}, \sigma}^{f}$ and $\varepsilon_{\beta, \mathbf{k}, \sigma}^{p}$ if their energy separation near the Fermi level is less then the effective coupling $\sim t^{\prime 2} / \varepsilon_{s}$ (mediated by the spacer modes at typical energy distance $\varepsilon_{s}$, see Fig. 3). Moreover, for the sake of clarity, we shall restrict the following consideration to the simplest situation of identical $f$ - and $p$-layers where all $n_{f}=n_{p}$ modes are relevant and can form resonant $f p$-pairs.

Thus, in the $\uparrow \uparrow$ configuration, there appears a strong hybridization in each $F^{\alpha}, P^{\alpha}$ pair, forming two collective 
modes as their bonding and anti-bonding combinations (in neglect of small contributions $\sim t^{\prime 2} /\left(\varepsilon_{s} \Delta\right) \ll 1$ of the rest of the modes):

$$
M_{j}^{\alpha, \pm} \approx \frac{1}{\sqrt{2}}\left\{\begin{array}{c}
F_{j}^{\alpha}, \quad \text { for } j \in J_{f}, \\
0, \quad \text { for } j \in J_{s}, \\
\pm P_{j}^{\alpha}, \quad \text { for } j \in J_{p},
\end{array}\right.
$$

where $J_{f, s, p}$ are the sets of atomic planes entering $f$-, $s$-, and $p$-layers (see inset in Fig. 3). The respective relaxation times are given by

$$
\begin{aligned}
& \left(\tau_{\alpha, \pm}^{\uparrow \uparrow}\right)^{-1} \approx \frac{\pi c V^{2}}{2 \hbar n} \sum_{\beta}^{\prime \prime} \rho_{\beta} \\
& \times\left(\sum_{j \in n_{f}}\left|F_{j}^{\alpha} F_{j}^{\beta}\right|^{2}+\sum_{j \in n_{p}}\left|P_{j}^{\alpha} P_{j}^{\beta}\right|^{2}\right) .
\end{aligned}
$$

Then we can use the exact sum rule for the amplitudes, Eq. 2,

$$
\sum_{j=1}^{n}\left(A_{j}^{\alpha} A_{j}^{\beta}\right)^{2}=\frac{1}{n+1}\left(1+\frac{\delta_{\alpha, \beta}+\delta_{\alpha, n+1-\beta}}{2}\right),
$$

to present the relaxation times, Eq. 10, as

$$
\tau_{\alpha, \pm}^{\uparrow \uparrow} \approx \frac{\hbar n}{2 \pi c V^{2} \sum_{\beta}^{\prime \prime} \rho_{\beta}} .
$$

Contrariwise, in the $\uparrow \downarrow$ configuration, all the relevant modes remain almost unhybridized, taking nearly "local" forms:

$$
\begin{aligned}
& M_{j}^{\alpha, f} \approx\left\{\begin{array}{l}
F_{j}^{\alpha}, \quad \text { for } \quad j \in J_{f}, \\
0, \quad \text { for } \quad j \in J_{s} \cup J_{p},
\end{array}\right. \\
& M_{j}^{\alpha, p} \approx \begin{cases}0, & \text { for } \quad j \in J_{f} \cup J_{s}, \\
P_{j}^{\alpha}, & \text { for } \quad j \in J_{p},\end{cases}
\end{aligned}
$$

and in this approximation we obtain for the relaxation times $\tau_{\alpha, i}^{\uparrow \downarrow}$ half the value of Eq. 12, Then the magnetoresistance, Eq. 8, is readily estimated as $\Delta R / R \approx 100 \%$. We notice that this result is practically independent of the parameters of interlayer coupling and impurity scattering, in particular it does not even need that lifetimes of majority and minority carriers be different (as necessary for quasiclassical regimes). The main MR effect

\begin{tabular}{|c|c|c|c|}
\hline \multicolumn{4}{|c|}{$1 \overline{C 1}^{-7} \times \Omega_{\alpha, \beta}(\mathrm{W})$} \\
\hline$\beta=1$ & $\beta=2$ & $\beta=3$ & $\beta=4$ \\
\hline$\alpha=1 \uparrow \uparrow 0.5175$ & 0.3431 & 0.3417 & 0.5151 \\
\hline$\uparrow \downarrow 1.0347$ & 0.6857 & 0.6824 & 1.0287 \\
\hline$\alpha=2 \uparrow \uparrow 0.3431$ & 0.5118 & 0.5097 & 0.3415 \\
\hline$\uparrow \downarrow 0.6857$ & 1.0224 & 1.0175 & 0.6818 \\
\hline$\alpha=3 \uparrow \uparrow 0.3417$ & 0.5097 & 0.5077 & 0.3402 \\
\hline$\uparrow \downarrow 0.6824$ & 1.0175 & 1.0127 & 0.6786 \\
\hline$\alpha=4 \uparrow \uparrow 0.5151$ & 0.3415 & 0.3402 & 0.5127 \\
\hline$\uparrow \downarrow 1.0287$ & 0.6818 & 0.6786 & 1.0228 \\
\hline
\end{tabular}
in the considered limit is due to the variation of coherent quantum states, induced by the relative rotation of magnetization of the FM layers.

\section{NUMERICAL CALCULATIONS}

To certify the above qualitative considerations, a detailed numerical calculation was done for the particular
Table I: Scattering factors $\left(\Omega_{\alpha, \beta}\right)$ for the Fermi modes $(\alpha, \beta=$ $1,2,3$ and 4$)$ in the parallel $(\uparrow \uparrow)$ and antiparallel $(\uparrow \downarrow)$ configurations.

\begin{tabular}{lcccc}
\hline \hline$\alpha$ & 1 & 2 & 3 & 4 \\
\hline $10^{19} \rho_{\alpha}\left(\mathrm{J}^{-1}\right)$ & 0.209 & 0.2439 & 0.3238 & 0.5517 \\
$10^{10}\left\langle v_{\alpha}^{2}\right\rangle\left(\mathrm{m}^{2} / \mathrm{s}^{2}\right)$ & 0.7431 & 2.2595 & 2.9334 & 2.6008 \\
$10^{-12} \tau_{\alpha}^{\uparrow \uparrow}(\mathbf{s})$ & 1.7018 & 1.8157 & 1.823 & 1.7094 \\
$10^{-12} \tau_{\alpha}^{\uparrow \downarrow}(\mathbf{s})$ & 0.8515 & 0.9091 & 0.9133 & 0.8563 \\
\hline \hline
\end{tabular}

Table II: The Fermi density of states $\rho_{\alpha}$, averages of squared Fermi velocities $\left\langle v_{\alpha}^{2}\right\rangle$ and relaxation times $\tau_{\alpha}$ for the Fermi modes $(\alpha=1,2,3$ and 4$)$ in the parallel $(\uparrow \uparrow)$ and antiparallel $(\uparrow \downarrow)$ configurations.

choice of parameters: $t=t^{\prime}=0.25 \mathrm{eV}, \Delta=0.5 \mathrm{eV}$, $\varepsilon_{s}=2 \mathrm{eV}$ (a single-band model for real $d$-bands of Co and $\mathrm{Cu}$ ), $n_{f}=n_{p}=4, n_{s}=3$ (a simple discrete structure of layers), and $V=0.5 \mathrm{eV}$, and $c=0.01$ (typical impurity parameters). The Fermi velocities (and their inverse values) for two characteristic directions in Brillouin zone were used to approximate the partial densities of states

$$
\rho_{\alpha} \approx \frac{a^{2} L_{\alpha}}{8 \pi 2 \hbar}\left(\frac{1}{v_{\alpha, \Gamma M}}+\frac{1}{v_{\alpha, M X}}\right)
$$

( $L_{\alpha}$ being the length of respective Fermi line, Fig. (4), and then $\left\langle v_{\alpha}^{2}\right\rangle \approx v_{\alpha, \Gamma M} v_{\alpha, M X}$. The obtained numerical results for $\Omega_{\alpha, \beta}, \rho_{\alpha}, \tau_{\alpha}$, and $\left\langle v_{\alpha}^{2}\right\rangle$ are illustrated in tables II and II respectively, for the $\uparrow \uparrow$ and $\uparrow \downarrow$ configurations. These numerical values lead to $\approx 99.65 \%$ of magnetoresistance, that is quite close to the maximum possible MR $=100 \%$ in the coherent regime. To compare with, for purely incoherent currents there will be no MR at all in such two-layer system, so that the finite effect only appears from their partial mixing due to scattering at the interfaces [6] and is estimated phenomenologically as $\sim \exp (-d / \ell)$ of the above maximum value.

Actually, the experimental MR values in specular spin 
valves [11, 12] are clearly lower than the above model estimates. This can be due to a number of important factors, not included into the present simple model (which therefore should be considered as a certain idealized reference case). First of all, the postulated ideal specularity condition (supposing the wave function fully confined within the $n$-plane system) cannot be exact in reality, and a considerable part of the electronic density can "escape" through the NOL barriers to adjacent non-magnetic (or AFM) layers. This part would act as a parallel conduction channel, mostly unchanged at reorientation of FM electrodes and hence restricting the magnetoresistive effect. Also, the used model of rigid Stoner shifts of spin subbands in FM electrodes of course overestimates sharpness of the spin-dependent energy barrier between these electrodes, where in fact the band structures are not uniform on scales of few atomic layers. Other restrictive factors are the temperature effect (by phonons and magnons), the roughness in the FM/NM interfaces and the presence of defects as grain boundaries, displacements and distortions in the crystalline structure, which all reduce the coherence of relevant quantum states and so the validity of the Landauer formula. At last, the single-band model may be oversimplified, compared to the real hybridized $s$ - $d$ band structures of transition metals used in various numerical studies of spin-valves [13, 14, 15], but it would be rather problematic to keep the analytic description at such detailed level. Nevertheless, a further development within the present model can be done, varying the number $n_{r}$ of relevant modes and admitting the presence of both spin polarizations among these modes.

\section{CONCLUSIONS}

A simple single-band tight-binding model was developed to estimate theoretically the maximum possible enhancement of GMR in the system of quantum coherent FM nanolayers, using a specific set of Boltzmann equations for spatially quantized and spin-resolved subbands and a Landauer-type formula for the spin-dependent conductance. It is shown that a limit GMR value close to $100 \%$ can be reached for a fully coherent (and fully specular) SSV nanostructure and the reducing factors for this value in real SSV systems are discussed.

\section{Acknowledgments}

This work was supported in part by FEDERPOCTI/0155, POCTI/CTM/45252/02 and POCTI/CTM $/ 59318 / 2004$ from Portuguese FCT and IST-2001-37334 NEXT MRAM projects. JMT and JV are thankful for FCT grants (SFRN/BD/24012/2005 and SFRN/BPD/2163/2005).
[1] B. Dieny, V. S. Speriosu, S. Metin, S. S. P. Parkin, B. A. Gurney, P. Baumgart, and D. R. Wilhoit, J. Appl. Phys, 69, 4774 (1991).

[2] M. N. Baibich, J. M. Broto, A. Fert, F. Nguyen Van Dau, F. Petroff, P. Eitenne, G. Creuzet, A. Friederich, and J. Chazelas, Phys. Rev. Lett. 61, 2472 (1988).

[3] A. Veloso, P. P. Freitas, P. Wei, N. P. Barradas, J. C. Soares, B. Almeida, and J. B. Sousa, Appl. Phys. Lett. 77, 1020 (2000).

[4] R. Zdyb and E. Bauer, Phys. Rev. Lett. 88, 166403 (2002).

[5] J. Graf, C. Jozwiak, A. K. Schmid, Z. Hussain, and A. Lanzara, Phys. Rev. B 71, 144429 (2005).

[6] R. E. Camley and J. Barnas, Phys. Rev. Lett. 63, 664 (1989).

[11] J. B. Sousa, J. O. Ventura, M. A. Salgueiro da Silva, P. P. Freitas, and A. Veloso, J. Appl. Phys. 91, 5321 (2002).

[12] V. Gehanno, P. P. Freitas, A. Veloso, J. Ferreira, B.
Almeida, J. B. Sousa, A. Kling, J. C. Soares, and M. F. da Silva, IEEE Trans. Magn., 35, 4361 (1999).

[9] D. Bagayoko, A. Ziegler, and J. Calloway, Phys. Rev. B 27, 7046 (1983).

[10] R. Landauer, IBM J. Res. Dev. 1, 223 (1957).

[11] J. B. Sousa, J. O. Ventura, M. A. Salgueiro da Silva, P. P. Freitas, and A. Veloso, J. Appl. Phys. 91, 5321 (2002).

[12] V. Gehanno, P. P. Freitas, A. Veloso, J. Ferreira, B. Almeida, J. B. Sousa, A. Kling, J. C. Soares, and M. F. da Silva, IEEE Trans. Magn., 35, 4361 (1999).

[13] E. Y. Tsymbal and D. G. Pettifor, J. Magn. Magn. Mater. 202, 163 (1999).

[14] J. Chen and J. Fernandez-de-Castro, J. Appl. Phys. 89, 6934 (2001).

[15] C. Blaas, L. Szunyogh, P. Wienberger, C. Sommers, P. M. Levy, and J. Shi, Phys. Rev. B 65, 134427 (2002). 